\title{
Anti-Ageing Cure
}

\author{
Nishant Patnaik \\ Varanasi, India \\ Email: patnaik639@yahoo.com
}

Received 21 February 2016; accepted 7 March 2016; published 10 March 2016

Copyright (C) 2016 by author and OALib.

This work is licensed under the Creative Commons Attribution International License (CC BY). http://creativecommons.org/licenses/by/4.0/

(c) $\underset{\mathrm{EY}}{\mathrm{i}}$ Open Access

\section{Abstract}

The anti-ageing drugs have become the pinnacle of increased focus on biotechnology and are linked with innovative clinical preventive drugs. The specialty is initiated on the claim of groundbreaking scientific and medical technologies. These innovative technologies aim to prevent, treat, and restrict age-related disorders. This research paper attempted to examine the various types of drugs and treatments methods for anti-ageing. One of the drugs for anti-ageing cure is rapamycin, which is a remedy that has the capacity to destroy the immune system. Calorie restriction is recommendable because it helps to prolong lifespan among ageing people. Another recommendable drug is metformin, which restores insulin sensitivity for people with type II diabetes. Antioxidants drugs are also crucial for prolonging lifespan. Hormonal therapies are effective treatment for prolonging lifespan of people. Human growth hormone, melatonin, estrogen, which is mostly for women, insulin-like growth factor, and DHEA are among the hormonal therapies vital for prolonging life.

\section{Keywords}

Anti-Ageing, Metformin, Diabetes, Antioxidants, Lifespan, Rapamycin, Hormones

\section{Subject Areas: Biochemistry, Neuroscience}

\section{Introduction}

Anti-ageing drugs have become the center of research interest in the recent decades. Anti-ageing cure is drugs that aim to prolong lifespan or reduce ageing among people. Even though age habitually confers wisdom, everyone wants to remain young in body and mind. The quest for people to maintain physical changes at haven has led to formation of huge pharmaceutical industries which aim to offer solutions to ageing. These industries manufacture medicines that increase cognition and help people to keep body's physical changes at bay. They promise moist skins, strength, and venous vitamins, which blend for energy. Regardless of this effort, it is not yet clear as to whether there is an effective anti-ageing cure since most of the current drugs are not anti-ageing. Therefore, this research paper attempts to examine the types of drugs and effective treatments suitable for anti- 
ageing.

\section{Anti-Ageing Drugs and Treatment}

Effective anti-ageing drugs are those that treat all diseases. In case a medicine is only fundamental for only one disease, that drug is not anti-ageing. Most of the current innovative drugs are not anti-ageing. For example, insulin rewards diabetes but it does not cure cancer. If it does not cure cancer, this means that insulin is not an antiageing drug. The studies indicate that the anti-ageing bill has been pushed as a bona fide drug for solving agerelated issues [1]. Scientists and health care providers want policy makers or other drug related funding agencies to consider drugs that delay age-related illness. The research study reveals that anti-ageing treatments have a physiological effect on ageing related diseases, as in [1]. The research on medical treatment for ageing has risen for the past few decades. Industries marketing drugs are now advertising drugs that prolong life. However, some medicines and lifestyles aiming to prolong life focus at physiological alleyways [2]. However, there are numerous products including drugs, supplements, and diets that have anti-ageing properties. Some of the famous antiageing drugs and treatment are listed as follows:

\subsection{Calorie Restriction (CR)}

CR helps to prolong lifespan of ageing people. CR is an effective anti-ageing intervention that delays human ageing. CR is the only known therapy commonly recommended because it does not have any side effect. CR such as restriction of food rich in fats and consumption of junky foods help to reduce diseases such as obesity. Poor food consumption can affect not only the health of people but also their age. In other words, unrestricted food consumption accelerates ageing diseases. CR requires reducing calorie intake while keeping a balanced diet at normal. Therefore, CR delays age-related diseases, and thus administering CR is an effective anti-ageing modality.

\subsection{Metformin}

Metformin is an oral antibiotic drug that restores insulin sensitivity for people with type II diabetes. It lowers breast cancer. It is an effective drug for cancer management. According to [3], metformin induces ovulation, especially to patients with polycystic ovary disorders. It is an effective drug for anti-ageing because it slows ageing and prolongs life span in rodents.

\subsection{Rapamycin}

Rapamycin is a drug that has the capacity to destroy the immune systems. Rapamycin acts on FK-binding protein 12, a substance that is usually recognized as immunophilin and it binds to immunosuppressive drugs [4]. A number of factors including glucose, insulin, and amino acid among others, stimulate the nutrient-sensing known as target of rapamycin (TOR). The TOR acts as an insulin receptor substrate. In this case, it obliterates this substrate when TOR pathway is stimulated, thereby contributing to insulin resistance. CR reduces the level of nutrients and insulin, which de-activates TOR. Like CR, rapamycin reduces fat cells and weight. The TOR slows down ageing in varied organisms. Rapamycin inhibits TOR; thus, a good medication for prolonging lifespan.

\subsection{Antioxidants}

Antioxidants drugs are effective for prolonging lifespan. The body has diverse antioxidant mechanisms, which defend itself from any damage that is caused by reactive oxygen species. The body has antioxidant enzymes that demand micronutrient co-factors including copper, manganese, and zinc among others for effective body activities. Inadequate intake of proper diet of these macronutrients will contribute to low antioxidant activities. Therefore, common antioxidants such as Vitamin A, Vitamin C and E, as well as coenzymes, are recommended as effective anti-ageing drugs [5]. Although there is little evidence on the ways these antioxidant drugs work, they are proven as effective ways for helping people to stay young. For example, in a mice experiment, the study found that the antioxidants do not slow ageing but can slightly augment longevity, as in [3].

Red wine was also found to be an effective product that acts as antioxidant vital for protecting brain ageing. 
Even though these products have been proven as effective antioxidants, there is lack of adequate research evidence on their impacts or side effects. However, in a mice experiment, one study found that antioxidants could accelerate development of cancer in mice, as in [3]. There is no proof that antioxidants delay ageing, but epidemiological research studies reveal that antioxidant supplements are likely to upturn mortality rate.

\subsection{Hormonal Therapies}

The hormonal level or imbalance usually slows down with age. As a result, many of the oldest treatment methods that still exist today are common anti-ageing therapy, which are based on the idea that hormonal imbalances or changes contribute to ageing. Reversing hormonal changes are imperative. The most common and widely acceptable treatment is using human growth hormone injections (hGH), as in [5]. hGH has been for many decades used as an effective anti-ageing cure. Research evidence shows that hGH ageing patients who have used this treatment feel younger and healthier, as in [5]. Despite the innovative hGH therapy, it has failed to live up to the present because of some side effects. One of the effects is that it contributes to diabetes, obesity due to weight gain, and high blood pressure. As the name, hGH implies stimulating growth. There have been a number of concerns as to whether this treatment stimulates cancer development in malignant tumor patients [6]. Another experiment conducted finds that hGH treatment in animals accelerates the lifespan in an old mice. For that reason, hGH can make aged patients to feel healthier and increase their lifespan.

There are also other hormones such as melatonin, estrogen, mostly for women, insulin-like growth factor (IGF-1), and DHEA among others, as in [5]. IGH-1 plays a significant role in ageing. Patients can purchase IGH-1 as a supplement, which is good for longevity. IGHF-1 injections can yield good results, but they are harmful; hence, the probability to extend lifespan is low. DHEA is crucial in therapy for ageing people in many ways. One of the ways is that it enhances immune system, restores memory, increases sexual appetite, and makes the skin smooth. Though it also reduces cancer risks, there is no clear scientific proof on this but minor side effects have been reported, as in [3]. Melatonin is also linked with life-extension of ageing people. Melatonin has some antioxidant functions of the brain, so it has some benefits to ageing patients. It delays the ageing process and is likely to protect the elderly people from age-related disorders. Lastly, estrogen is a good anti-ageing treatment, especially for women. It is done in combination with hormone replacement psychotherapy since it helps in reducing menopause in women.

\section{References}

[1] Hayden, C.E. (2015) Anti-Ageing Pill Pushed as Bona Fide Drug. Nature.com. http://www.nature.com/news/anti-ageing-pill-pushed-as-bona-fide-drug-1.17769

[2] Longo, V.D., Antebi, A., Bartke, A., et al. (2015) Interventions to Slow Ageing in Humans: Are We Ready? Ageing Cell, 14, 497-510. http://dx.doi.org/10.1111/acel.12338

[3] Blagosklonny, M.V. (2009) Validation of Anti-Ageing Drugs by Treating Age-Related Diseases. Ageing, 1, $281-288$.

[4] Martínez-Sanchis, S., Bernal, M.C., Montagud, J.V., et al. (2011) Effects of Immunosuppressive Drugs on the Cognitive Functioning of Renal Transplant Recipients: A Pilot Study. Journal of Clinical \& Experimental Neuropsychology, 33, 1016-1024. http://dx.doi.org/10.1080/13803395.2011.595396

[5] Pedro de Magalhaes, J. (2014) Anti-Ageing Medicine. Senescence.info. http://www.senescence.info/antiageing_science.html\#Antioxidants

[6] Manosroi, A., Chutoprapat, R., Abe, M., et al. (2012) Anti-Ageing Efficacy of Topical Formulations Containing Niosomes Entrapped with Rice Bran Bioactive Compounds. Pharmaceutical Biology, 50, 208-224. http://dx.doi.org/10.3109/13880209.2011.596206 